\title{
Respirators, Face Masks, and Diverse Populations: An Analysis of 3D Facial Anthropometrics
}

\author{
Kayna HOBBS-MURPHY *1, Kristen MORRIS ${ }^{2}$, Bill BRAZILE ${ }^{1}$, John ROSECRANCE ${ }^{1}$ \\ ${ }^{1}$ Department of Environmental and Radiological Sciences, College of Veterinary Medicine and \\ Biomedical Sciences, Colorado State University, Fort Collins CO, USA; \\ 2 Department of Design and Merchandising, College of Health and Human Sciences, \\ Colorado State University, Fort Collins CO, USA
}

https://doi.org/10.15221/21.60

\section{Introduction}

Facial coverings have become a topic of research concern in recent years, partially due to the COVID19 pandemic. Though cloth face masks and disposable surgical face masks are effective in the context of viral infections, US National Institute for Occupational Safety and Health (NIOSH)-certified N95 respirators are considered to be the best mask for use by healthcare workers "during procedures to protect both the patient and the health care personnel from the transfer of microorganisms, body fluids, and particulate matter", and may be best for protection against viral particulates [1]. Furthermore, N95 respirators (also known as quarter-mask respirators) as well as other half-mask or full-facepiece respirators are common for use in occupations where the worker is exposed to dust, silica, and other small particulates. All three types of respirators can be seen in Figure 1. Though filtering ability is an important factor for respirator protection, the 'seal' of the respirator on the face is also considered to be a key factor in how much protection is provided to the wearer [2]. A common source of reduced protection against viral and other particulate exposures for surgical and cloth face masks is air leakage along the parameter of the mask. Thus, having a more fitted 'seal' incorporated into these non-respirator masks may improve protection for wearers [3,4].

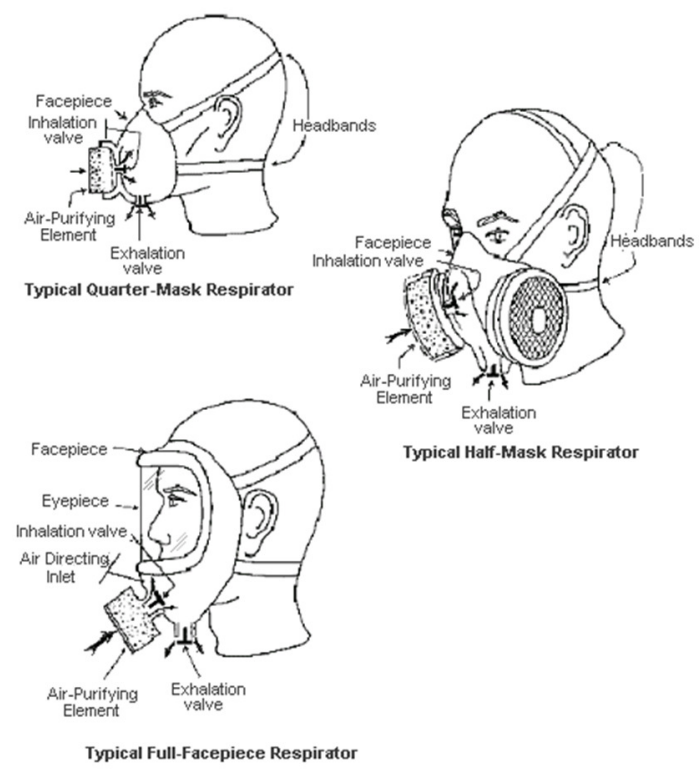

Fig. 1: Three types of respirators: Quarter-mask (N95), half-mask, and full-facepiece. Note: Quarter-mask respirators without exhalation valves should be used in viral particulate settings. Source: OSHA.gov [5].

This research is being conducted to quantify the differences in 3D facial dimensions among a sample $(n=2022)$ of US workers from a variety of racial / ethnic backgrounds and demographic characteristics using facial scans (collected by Human Solutions). A secondary objective is to assess inter- and intrarater reliabilities of 3D-based measurement collection methods. Results of this research have implications for respirator and face mask design and fit as well as utilization of $3 D$ scanning as an accurate anthropometric tool for measurement collection. Relevant literature regarding previous research, employment trends for various demographics, manual measurements vs 3D scanning, and intra-/inter-rater reliability in 3D measurement collection are discussed, followed by methods for data collection and analysis, as well as implications and limitations for this on-going research.

*corresponding author: Kayna.Hobbs-Murphy@colostate.edu 


\subsection{Previous facial anthropometry research and surveys}

A wide array of research has investigated how demographic differences correlate to differences in face shape and size, or facial anthropometrics, specifically in terms of respirator seal and/or respirator fit (these terms are often used interchangeably). Brazile et al. (1998) found evidence of significant differences in facial anthropometric measures between males and females as well as between "white, African-American, and Mexican-American" participants [6]. The researchers concluded that individual differences in nose breadth and nose protrusion were significant in terms of respirator fit, and "facial dimensions based on gender, race/ethnicity, and the interaction of the two were not" (p.233) [6]. Similarly, Yokota et al., 2005, found that facial anthropometrics of US male soldiers of White, Black, and 'mixed' race/ethnicity fell into a normal distribution of a single-race group via canonical discriminant function analysis [7]. Zhuang et al. (2010) found significant differences in facial anthropometrics across gender, race/ethnicity (four groups), as well as age (three groups) and occupation (six groups); these differences were considered significant for design of respirators and other protective head equipment (such as helmets) [8]. The lack of inclusion of Asian peoples in the previous studies is a stated weakness of each. Other research has focused specifically on Asian facial anthropometrics: one study found that products designed for US white males are not suitable for fitting Korean male and female pilots [9], and another found that female Chinese faces are significantly different in width and length than all other demographics' faces [10]. A current weakness in the literature is the lack of a fully diverse test population, which includes both male and female gendered participants, White/Caucasian (White for brevity), Black/African/African American (Black for brevity), LatinX/Latino/Latina/Hispanic (LatinX for brevity), and Asian/Asian-American (Asian for brevity) participants, and participants of all ages.

The U.S. government has invested considerable resources into various anthropometric surveys throughout history: two Anthropometric Surveys (abbreviated as ANSUR I and ANSUR II) have been conducted by the U.S. Army in 1988 and 2012 to gather whole-body anthropometric information of 26,000 (ANSUR I) and 12,000 (ANSUR II) army personnel, including soldiers and civilian employees $[11,12]$. Though these surveys are comprehensive and have provided invaluable standards for rigorous collection of anthropometric data, their generalizability is of question, as most soldiers are of a young age, of male gender, and perhaps not representative of the working population in terms of race/ethnicity. Of specific interest for respirator fit are two surveys done by NIOSH: a 1973 Los Alamos National Laboratory (LANL) respirator fit-test panel and a $2007 \mathrm{NIOSH}$-sponsored anthropometric survey of U.S. respirator users [13], [14]. Though these surveys are specific to respirators, their representation of the current U.S. working population is lacking; Yang et al. (2007) note that Chinese and Asian populations, known to have different facial anthropometry, are not well represented in these surveys [15]. Furthermore, NIOSH's 2007 survey, conducted by Anthrotech, offered many suggestions for improvement in future surveys [14]. Of note for the study at hand are the suggestions from NIOSH to ensure study sample populations are representative (in terms of demographics) of the current working population, consider use of 3D scan data, and analyze measurement error (intra- and inter-rater reliability) [14].

\subsection{Current employment trends for various demographics}

According to U.S. Census Bureau Data from 2020 in which near 150,000,000 employed workers over 16 years of age were accounted for, the demographics of workers were (at the time, in the sample) $78 \%$ White, $17.6 \%$ LatinX, $12.1 \%$ Black, and $6.4 \%$ Asian (totals do not equal $100 \%$ due to missing data, and LatinX ethnic status can be present with any race status) [16]. Of these workers, $46.8 \%$ were women (use of the word 'women' versus 'female' is specific to how these data are presented) [16]. Per the suggestion from $\mathrm{NIOSH}$ to ensure study populations for facial anthropometric research are representative of the current working population, a comparison of these percentages and the percentages of our sample will be presented in Section 2: Methods.

The U.S. Census Bureau Data from 2020 are presented in the context of percent of total employed per demographic group per occupation (LatinX, Black, Asian, Women, and White). Data were imported into $R$ and analyzed (version 4.0.5) for occupations with the highest percentage of workers for all demographics except White (Figure 2). Occupations with high percentages of LatinX workers are occupations that likely require regular respirator wear for particulates (per Occupational Safety and Health Administration standard 1910.134), and include drywall and ceiling install, insulation work, cement work, carpet work and flooring, roofing, painting, agricultural work, housekeeping, construction, and landscaping [16]. Occupations with high percentages of Black workers may require the use of N95 quarter-mask respirators for viral protection, as the occupations are social in nature: postal service, 
healthcare, transportation, parking, correctional facility, and security [16]. Asian workers make up a large percentage of manicurists and pedicurists, as well as higher qualification jobs in various sciencebased fields, suggesting that N95 quarter-mask respirators would be necessary for both particulate and viral/social purposes for this demographic [16]. Lastly, women/females make up a large percentage of teachers and healthcare workers, which also suggests the potential for use of N95 quarter-mask respirators for viral protection in social environments [16]. Given that each demographic makes up a large percentage of occupations in which some type of respiratory protection may be needed, the present research will be valuable in providing comparative anthropometric data for respirator sizing and fit outcomes, and ideally provide better occupational protection to these workers as an outcome.

Occupations with highest $\%$ of LatinX workers 2020 US Bureau of Labor Statistics Data

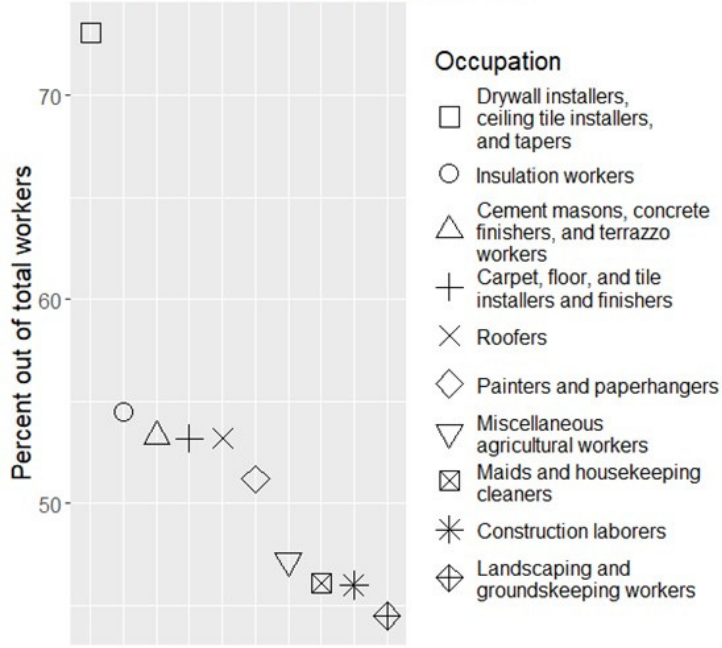

Occupations with highest \% of Asian workers 2020 US Bureau of Labor Statistics Data

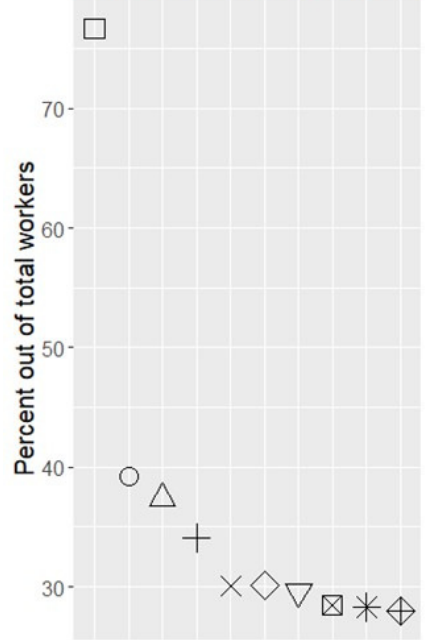

Occupations with highest \% of Black workers 2020 US Bureau of Labor Statistics Data

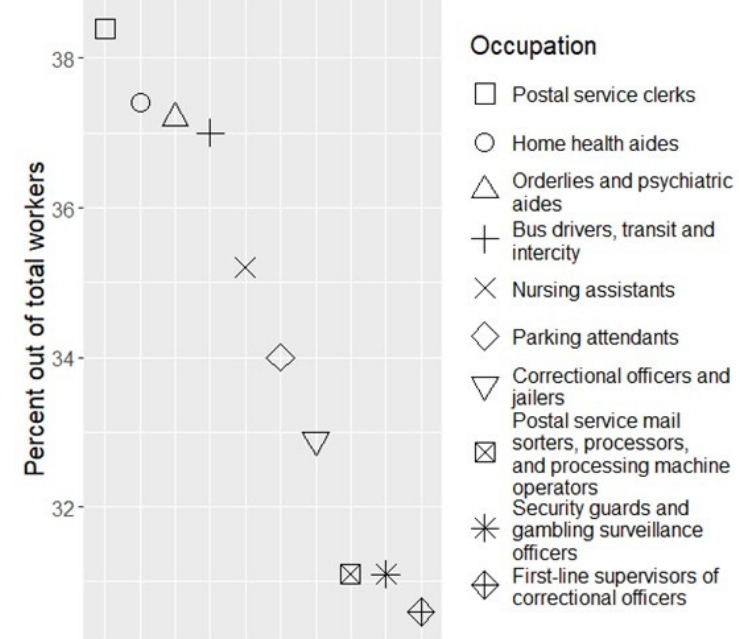

Occupations with highest \% of Women workers 2020 US Bureau of Labor Statistics Data

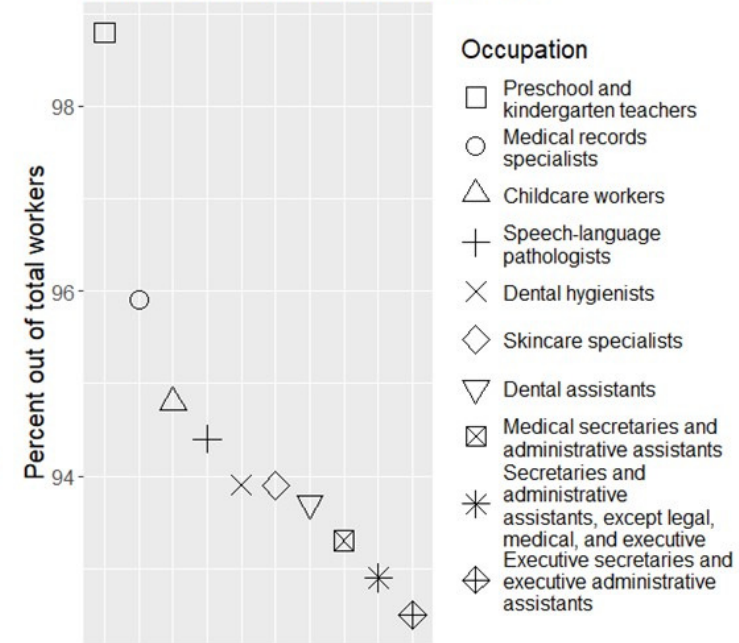

Fig. 2: Occupations with highest percentage of LatinX, Black, Asian, and women/female workers [16].

\subsection{Manual measurements versus 3D scanning of facial anthropometrics}

3D scanning is "a technology for creating high-precision 3D models of real-world objects" [17]. 3D scanners collect surface data from an object by capturing the geometry versus a 2D image; 3D scans are made up of triangles or polygons that "replicate" the scanned object in "minute detail" [17]. Though 3D scanning has a long list of potential applications, of particular focus is its use in gathering anthropometric data for design applications (medical devices, wearable products, and animation, to name a few). Prior to the use of 3D body scanning, manual measurements were completed by a trained expert; today, experts are continually realizing benefits of non-contact measurement methods, but often 
still question the validity of 3D scanning [18]. A common question regarding 3D scanning is whether the 3D scanned object, or body for anthropometric work, is accurately representative of the original object or body in the real world. Many studies and meta-analyses have been done to address these concerns, for many different types of 3D scan systems. Of specific concern for this work is the validity of the Artec Eva scanner, as data for this research was collected via this hand-held system. Unfortunately, very little work has been done to validate the Artec Eva scanner in terms of accuracy, as has been seen in the literature for other scanning systems.

Previous applied work has used the Artec Eva scanner for development of custom 3D printed half-mask facepieces to provide an air-tight seal between the face and respirator; this work found that 3D scanning constituted a "viable option" for custom 3D printing of these facepieces, and that the fit of the face pieces did create an air-tight seal with positive wearer assessments [19]. In another study, Artec Eva was compared to the Romer scanner (considered criterion standard) for measurement of residual lowerlimb models; the researchers concluded that the Artec Eva scanner was a valid tool for data collection in this context, with the mean percentage error (1.4\%) falling within the acceptable range for "current scanning technologies" [20]. Facial surgery research concluded that Artec Eva's validity in data collection is "more accurate" than a different scanning system called FaceScan3D, but both scanners' accuracies were within the "same range" as other commercially available 3D scanners [21].

A weakness that exists in the literature is the lack of comparison between manually collected facial measurements and 3D collected facial scans and subsequent measurements. One study in the field of craniofacial anthropometry addresses this weakness: measurements were gathered both manually on the face, and digitally from the Artec Eva scanner [22]. The researchers found that measurements obtained by the Artec Eva "showed excellent reliability and accuracy" compared to measurements gathered manually [22]. More research should be done to validate the accuracy of the Artec Eva for facial anthropometry data collection as compared to manual measurement data collection. In the present work, data were purchased from Human Solutions, and manual measurements were not collected. However, based on the current literature, Artec Eva's internal certification of $0.1 \mathrm{~mm}$ accuracy, and the comparative analysis nature (between demographics) of this work, use of 3D facial scan data collected by the Artec Eva was deemed to be suitable [23].

\subsection{Inter- and intra-rater reliability in 3D measurement collection}

In anthropometric studies, landmarking must be done by either a human or a program to set locations for where body measurements are to be gathered. In manual measurement collection, the measurer is able to palpate the body for bony landmarks, which aids in precision of both landmarking and the subsequent measurement. Because 3D scan objects exist virtually and statically, palpation is not possible. Scan subjects with protrusive landmarks, such as a strong brow line or prominent cheekbones, may be relatively simple to place landmarks upon. However, many people do not have such prominent landmarks that allow for visual landmarking without palpation. Thus, the issue of landmarking on a nonpalpable 3D object poses potential limitations in terms of data reliability. Inter- and intra-rater reliability scoring is a common way to address reliability concerns in clinical research (which includes anthropometric research) [24]. Kouchi and Mochimaru (2011) maintain that these reliabilities are necessary for evaluation of landmarking errors in 3D anthropometry specifically, as many former research efforts have evaluated validity of measurements only [25]. These researchers compared the inter- and intra-rater reliabilities of body landmarking on a 3D body scan, done by an expert anthropometrist and a novice measurer, finding that intra-rater reliability was lower for the expert and inter-rater reliability was not within the acceptable range as outlined by authors of the U.S. Army ANSUR I survey $[25,26]$. In the present work, inter- and intra-rater reliability will be thoroughly investigated. Elaboration of methodology for these reliabilities is found within the subsequent outline of methods.

\section{Methods}

Data for this research were purchased from Human Solutions, who collected the data from 2020-2021. Each data collection participant $(n=2022)$ self-reported on demographic variables of age, race/ethnicity, and gender prior to scanning. Figure 3 shows the count and percentage of each race/ethnicity, in order of most prominent to least prominent within the sample. $61.5 \%$ of participants self-identified as White, $27.1 \%$ as Black, $4.9 \%$ as LatinX, and $4.6 \%$ as Asian. This sample appears to be somewhat adequate in representing the current workforce, which according to the US. Bureau of Labor Statistics was $78 \%$ White, 12.1\% Black, 17.6\% LatinX, and 6.4\% Asian in 2020 [16]. Statistical analysis will be done to 
adjust for discrepancies in the percent per race/ethnicity in the data sample populations compared to the 2020 employed persons population. Data is not given in by the US Bureau of Labor Statistics for American Indian, Alaska Native, Native Hawaiian, or Other Pacific Islander workers nor for Non-Binary gender $(0.25 \%$ of sample) [16]. Due to small sample size and lack of information about these demographics' occupations, these data will be omitted from analysis $(n=1965)$. Figure 4 shows the age and gender frequencies within each of the top four racial/ethnic groups (variable y axis count); White, LatinX, and Asian participants tended to be younger, while Black participants' ages were more varied. The sample is $53.1 \%$ female, and $46.9 \%$ male.

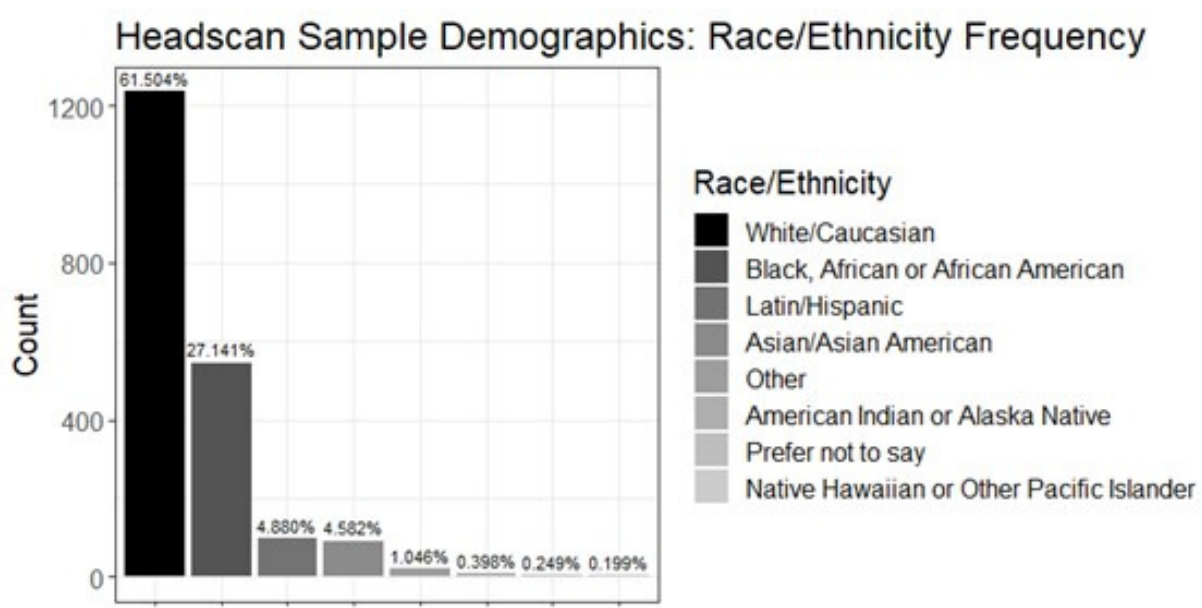

Fig. 3: Race/Ethnicity makeup of sample ( $n=2022)$.

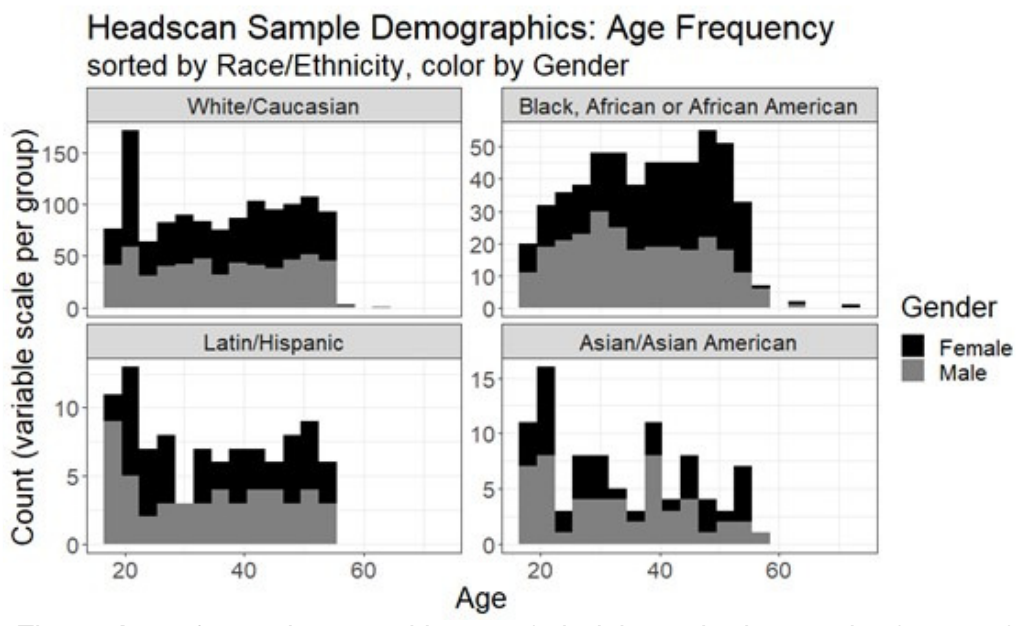

Fig. 4: Age of sample, sorted by race/ethnicity, color by gender $(n=1965)$.

After each scan participant reported on their demographics, a 3D head scan was taken by Human Solutions using the Artec Eva 3D scanner. Hair was covered and compressed using a swimmer's cap to better capture scalp shape and not occlude measurements that include head circumference. Figure 5 shows an example view of the 3D scans gathered from each participant. On each 3D scan, landmarks from Figure 6 will be placed using the Anthroscan tool from Human Solutions. To assess inter- and intra-rater reliability, all landmark-placing staff will be assigned to landmark an identical subset of 3D scans three times; from this, it will be possible to gather information regarding how reliably landmarks are being placed by each staff member and between all staff members. After landmark placement is complete, Anthroscan will automatically output the measures seen also in Figure 6. All landmarks and measures used in this work were developed using ANSUR studies, NIOSH respirator studies, and respirator fit expert input (Figure 6) [11,12,26,27]. 
Proceedings of 3DBODY.TECH 2021

12th Int. Conference and Exhibition on 3D Body Scanning and Processing Technologies, 19-20 Oct. 2021, Lugano, Switzerland

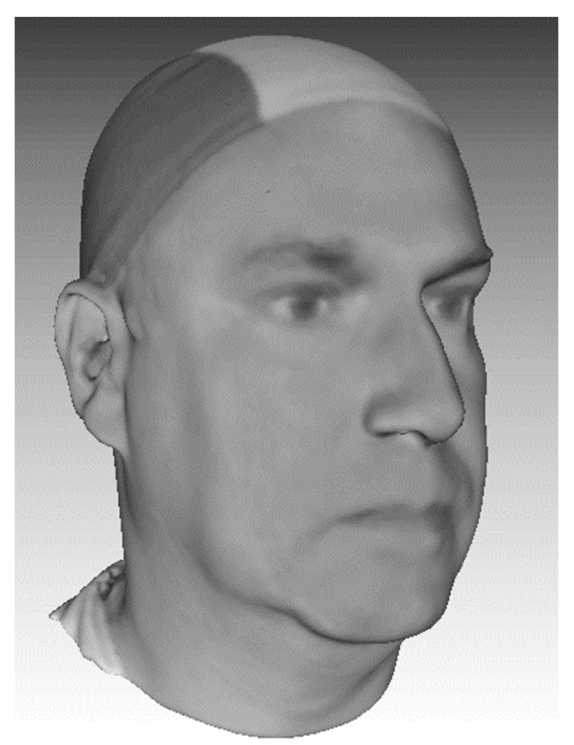

Fig. 5: Side front view of 3D head scan from Human Solutions (collected with Artec Eva 3D scanner).
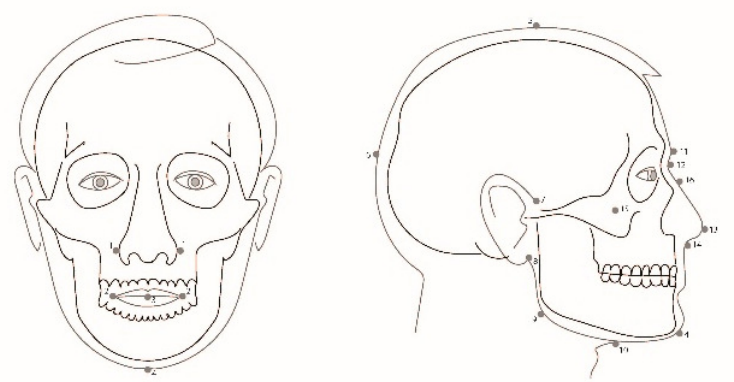

Landmarks

1) Alare

2) Cheillion

3) Stomion

4) Menton

5) Top of head

6) Opisthocranion

7) Tragion

8) Earlobe juncture

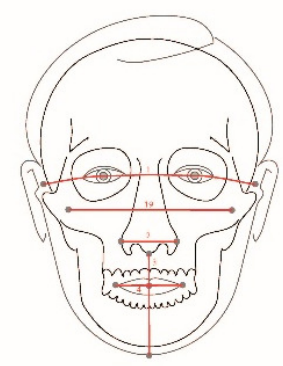

(\#: contour and linear, illustrated as contour only)

1) Tragion-Tragion \#

2) Alare-Alare

11) Tragion-Subnasale

3) Subnasale-Menton

4) Cheillion-Cheillion

5) Top of head-Tragion

6) Back of head-Glabella

7) Tragion-Earlobe juncture

8) Tragion-Gonion

10) Submandibular

11) Glabella

12) Sellion

13) Pronasale

14) Subnasale

15) Zygomatic arch

16) Dorsal hump

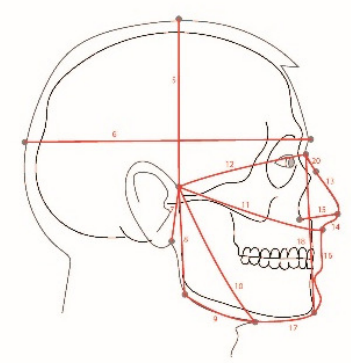

12) Tragion-Sellion

13) Sellion-Pronasale \#

14) Pronasale-Subnasale \#

15) Pronasale-Alare \#

16) Subnasale-Menton \#

17) Submandibular-Menton

18) Sellion-Menton (linear)

19) Bizygomatic Width \#

20) Sellion-Dorsal Hump

Fig. 6: Face landmarks and measures for data analysis. 
After all measures from the $n=1965$ sample have been gathered and compiled into a dataset, the data will be analyzed using multiple linear regression, principal component analysis, and clustering methods in R statistical software [28]. Inter- and intra-rater reliabilities will be held to a standard of $75 \%$ reliability (strong agreement) using Cohen's kappa coefficient [29]. The results of analyses will make it possible to provide design, sizing, and fit suggestions to mask manufacturers for workers based on ethnicity, race, and gender.

\section{Implications and Limitations}

One limitation of this work is the lack of expert-gathered manual anthropometric measurements to quantify differences in manual versus $3 \mathrm{D}$ data collection methods. We encourage future researchers to continue to address the pros and cons of $3 \mathrm{D}$ scanning versus manual measurement collection, specifically for facial anthropometry and using the Artec Eva 3D scanner.

This research seeks to understand how face shape and size differ across ethnic/racial populations, age groups, and gender to provide useful information for respirator design, sizing, and fit. Previous research in facial anthropometrics for respirator outcomes has not utilized 3D scan data, nor has the research seen a sample that is representative of the diversity seen in the workforce. For these reasons, this work is expected to fill a gap within the literature. Furthermore, reporting of inter- and intra-rater reliabilities will allow for better understanding of the reliability of utilizing 3D scan data in future facial anthropometric work. 3D scanning continues to become a strong tool for anthropometric data collection, and should continue to be investigated in these contexts.

\section{References:}

[1] US Food and Drug Administration, "N95 Respirators, Surgical Masks, Face Masks, and Barrier Face Coverings," Sep. 15, 2021. https://www.fda.gov/medical-devices/personal-protectiveequipment-infection-control/n95-respirators-surgical-masks-face-masks-and-barrier-facecoverings (accessed Sep. 23, 2021).

[2] CDC, Niosh, and NPPTL, "DHHS (NIOSH) Publication No. 2018-130, Filtering out Confusion: Frequently Asked Questions about Respiratory Protection, User Seal Check", doi: 10.26616/NIOSHPUB2018130.

[3] A. Konda, A. Prakash, G. A. Moss, M. Schmoldt, G. D. Grant, and S. Guha, "Aerosol Filtration Efficiency of Common Fabrics Used in Respiratory Cloth Masks," ACS Nano, vol. 14, pp. 6339-6347, 2020, doi: 10.1021/acsnano.0c03252.

[4] A. Konda, A. Prakash, G. Moss, M. Schmoldt, G. Grant, and S. Guha, "Correction to Aerosol Filtration Efficiency of Common Fabrics Used in Respiratory Cloth Masks," ACS Nano, vol. 2020, no. 5, pp. 6339-6347, 2020, doi: 10.1021/acsnano.0c03252.

[5] "OSHA Technical Manual (OTM) - Section VIII: Chapter 2 | Occupational Safety and Health Administration." https://www.osha.gov/otm/section-8-ppe/chapter-2\#intro (accessed Sep. 14, 2021).

[6] W. J. Brazile, R. M. Buchan, D. R. Sandfort, W. Melvin, J. A. Johnson, and M. Charney, "Respirator Fit and Facial Dimensions of Two Minority Groups," Applied Occupational and Environmental Hygiene, vol. 13, no. 4, pp. 233-237, 1998, doi: 10.1080/1047322X.1998.10390073.

[7] M. Yokota, "Head and facial anthropometry of mixed-race US Army male soldiers for military design and sizing: A pilot study," Applied Ergonomics, vol. 36, pp. 379-383, 2005, doi: 10.1016/j.apergo.2005.01.009.

[8] Z. Zhuang, D. Landsittel, S. Benson, R. Roberge, and R. Shaffer, "Facial Anthropometric Differences among Gender, Ethnicity, and Age Groups," Ann. Occup. Hyg, vol. 54, no. 4, pp. 391-402, 2010, doi: 10.1093/annhyg/meq007.

[9] W. Lee et al., "Analysis of the facial anthropometric data of Korean pilots for oxygen mask design," in Proceedings of the Human Factors and Ergonomics Society, 2012, pp. 1927-1931. doi: $10.1177 / 1071181312561282$.

[10] Y. Luximon, R. Ball, and L. Justice, "THE CHINESE FACE: A 3D ANTHROPOMETRIC ANALYSIS." [Online]. Available: www.rapidform.com 
[11] C. C. Gordon et al., "Anthropometric survey of US army personnel: methods and summary statistics 1988.," Yellow Springs OH, 1989.

[12] C. C. Gordon et al., "2012 anthropometric survey of U.S. Army personnel: methods and summary statistics," Natick MA, 2014.

[13] A. Hack, E. C. Hyatt, B. J. Held, T. 0 Moore, C. P. Richards, and J. T. Mcconville, "Selection of Respirator Test Panels Representative of U.S. Adult Facial Sizes (LA-5488)," Los Alamos, New Mexico, USA, Dec. 1973.

[14] J. C. Bailar, E. A. Meyer, and R. Pool, Assessment of the NIOSH head-and-face anthropometric survey of U.S. respirator users. National Academies Press, 2007. doi: 10.17226/11815.

[15] L. Yang, H. Shen, and G. Wu, "Racial differences in respirator fit testing: A pilot study of whether American fit panels are representative of Chinese faces," Annals of Occupational Hygiene, vol. 51, no. 4, pp. 415-421, 2007, doi: 10.1093/annhyg/mem005.

[16] U.S. Bureau of Labor Statistics, "2020 Annual Averages - Employed persons by detailed occupation, sex, race, and Hispanic or Latino ethnicity." https://www.bls.gov/cps/cpsaat11.htm (accessed Sep. 14, 2021).

[17] N. Kivolya, "What are 3D scanners used for?" https://www.artec3d.com/learning-center/whatare-3d-scanners-used-for (accessed Sep. 17, 2021).

[18] S. B. Heymsfield, B. Bourgeois, B. K. Ng, M. J. Sommer, X. Li, and J. A. Shepherd, "Digital anthropometry: a critical review," European Journal of Clinical Nutrition, vol. 72, pp. 680-687, 2018, doi: 10.1038/s41430-018-0145-7.

[19] K. Makowski and M. Okrasa, "Application of 3D scanning and 3D printing for designing and fabricating customized half-mask facepieces: A pilot study," Work, vol. 63, no. 1, pp. 125-135, 2019, doi: 10.3233/WOR-192913.

[20] E. Seminati, D. C. Talamas, M. Young, M. Twiste, V. Dhokia, and J. L. J. Bilzon, "Validity and reliability of a novel 3D scanner for assessment of the shape and volume of amputees' residual limb models," 2017, doi: 10.1371/journal.pone.0184498.

[21] A. Modabber et al., "Evaluation of the accuracy of a mobile and a stationary system for threedimensional facial scanning," 2016, doi: 10.1016/j.jcms.2016.08.008.

[22] C. Franco, S. Gomes, M. R. Libdy, and D. Normando, "Scan time, reliability and accuracy of craniofacial measurements using a 3D light scanner is," 2019, doi: 10.1016/j.jobcr.2019.07.001.

[23] "3D Object Scanner Artec Eva | Best Structured-light 3D Scanning Device." https://www.artec3d.com/portable-3d-scanners/artec-eva-v2 (accessed Sep. 17, 2021).

[24] F. B. Underwood, "Clinical research and data analysis," in Orthopaedic Physical Therapy Secrets, Third., J. D. Placzek and D. A. Boyce, Eds. Elsevier, 2017, pp. 150-162. doi: 10.1016/B978-0-323-28683-1.00020-5.

[25] M. Kouchi and M. Mochimaru, "Errors in landmarking and the evaluation of the accuracy of traditional and 3D anthropometry," Applied Ergonomics, vol. 42, no. 3, pp. 518-527, 2011, doi: 10.1016/j.apergo.2010.09.011.

[26] C. Clauser, I. Tebbetts, B. Bredtmiller, J. McConville, and C. C. Gordon, "Measurer's handbook: U.S. Army anthropometric survey 1987-1988," Yellow Springs, OH, 1988.

[27] Z. Zhuang, B. Bradtmiller, and R. E. Shaffer, "New respirator fit test panels representing the current U.S. civilian work force," Journal of Occupational and Environmental Hygiene, vol. 4, no. 9, pp. 647-659, Sep. 2007, doi: 10.1080/15459620701497538.

[28] G. Vinué, "Anthropometry: An R Package for Analysis of Anthropometric Data", Accessed: Sep. 23, 2021. [Online]. Available: http://www.uv.es/vivigui/docs/biclustDepth.

[29] M. L. McHugh, "Interrater reliability: the kappa statistic," Biochemia medica, vol. 22, no. 3, pp. 276-282, 2012. 